\title{
In silico study of morphine-like effects of ethanol intake: docking of acetaldehyde conjugates with monoamines to the mu-opioid receptor
}

\begin{abstract}
Acetaldehyde produced after the ethanol intake forms conjugates with monoamines. Those conjugates are thought to be able to bind mu-opioid receptors and cause morphine-like effects. The aim of this study was to check the ability of acetaldehyde conjugates with dopamine (salsolinol), serotonin, tryptamine and tryptophan, as well as their enantiomers and derivatives to bind mu-opioid receptor structure available in the Protein Data Bank (www.pdb.org) using the Docking Server (www.dockingserver. com) and the FlexX method. The results showed that N-methyl-R-salsolinol, unlike its stereoisomer, is the most potent agonist of the mu-opioid receptor. The strength of morphine-like effect observed after alcohol intake should directly depend on the rate of R-salsolinol formation and N-methylation, but it should show an inverse dependence on the rate of N-methyl-R-salsolinol dehydrogenation. It was found that an $\mathrm{N}$-methylated and dehydrogenated derivative of the acetaldehyde conjugate with tryptophan was another potent agonist of the mu-opioid receptor. Other compounds checked in this study were shown to act as antagonists and not agonists of the muopioid receptor.
\end{abstract}

Keywords: mu-opioid receptor, molecular docking, ethanol, acetaldehyde, n-methyl-r-salsolinol
Volume 4 Issue 3 - 2016

\author{
Khrustalev Vladislav Victorovich,' Lelevich \\ Sergey Vladimirovich, ${ }^{2}$ Khrustaleva Tatyana \\ Aleksandrovna, ${ }^{3}$ Skorobogatiy Maksim \\ Vyacheslavovich,' Demenchuck Ekaterina \\ Aleksandrovna' \\ 'Department of General Chemistry, Belarusian State Medical \\ University, Belarus \\ 2Department of Clinical Laboratory Diagnostics, Allergology and \\ Immunology, Grodno State Medical University, Belarus \\ ${ }^{3}$ Laboratory of Cellular Technologies, Institute of Physiology of \\ the National Academy of Sciences of Belarus, Belarus
}

Correspondence: Khrustalev VV, Belarusian State Medical University, Dzerzinskogo 83, Minsk, Belarus, Tel +375172725295, Emailvvkhrustalev@mail.ru

Received: October 05, 2016 | Published: November 08, 2016

\section{Introduction}

Addiction to alcohol is one of the main problems in society. Currently, several biochemical mechanisms underlying the development of alcoholism have been discovered. Ethanol $\left(\mathrm{C}_{2} \mathrm{H}_{5} \mathrm{OH}\right)$ was shown to be able to interact directly with several receptors on neural cells (nicotinic acetylcholine receptors, ${ }^{1} \mathrm{G}$ protein-gated inwardly rectifying $\mathrm{K}^{+}$channels, ${ }^{2}$ glycine receptors, ${ }^{3}$ gamma-aminobutyric acid receptors, ${ }^{4} \mathrm{~N}$-methyl-D-aspartate receptors). ${ }^{5}$ Consequences of such binding are thought to cause mental effects of a low dose alcohol intake (such as excitation and anxiolytic effect). Of course, ethanol should also be able to interact with many other receptors and even enzymes ${ }^{6,7}$ contributing to the mental effects development. Ethanol is metabolized in cells by alcohol dehydrogenases. The product of ethanol oxidation by that family of enzymes (acetaldehyde $-\mathrm{CH}_{3} \mathrm{CHO}$ ) is more reactive than ethanol itself. It is known that acetaldehyde can form conjugates with biogenic amines (with dopamine, serotonin, tryptamine, and tryptophan) both spontaneously and with the help of enzymes. ${ }^{89}$ Such conjugation results in the formation of an additional ring. Conjugates of abovementioned biogenic amines and acetaldehyde demonstrate some structural similarities with morphine. ${ }^{10}$ For this reason, they were thought to bind mu-opioid receptors and cause morphine-like effects after high dose alcohol intake (stimulation, reinforcement, and then sedation, loss of consciousness, and amnesia). ${ }^{11}$

The most studied conjugate of acetaldehyde and biogenic amine is known under the name salsolinol. ${ }^{8}$ That compound (1-methyl6,7-dihydroxy-1,2,3,4-tetrahydroisoquinoline) can be produced from dopamine and acetaldehyde. Interestingly, salsolinol is naturally produced in neural tissue and, probably, plays some role in neurotransmission. ${ }^{8}$ During the high dose alcohol intake, its concentration grows to a level that is much higher than the background one. ${ }^{11}$ Biodegradation of salsolinol includes at least two steps. First, salsolinol is methylated by $\mathrm{N}$-methyltransferase (the product is 1,2-dimethyl-6,7-dihydroxy-1,3,4-trihydroisoquinoline). ${ }^{12}$ Then $\mathrm{N}$-methyl-salsolinol is oxidized by amine oxidase and forms 1,2-dimethyl-6,7-dihydroxyisoquinolinium ion. ${ }^{12}$

In numerous studies, salsolinol was shown to promote motor activity in rats. That motor activity is inhibited by mu-opioid receptors antagonists. ${ }^{8,11,12}$ Moreover, rats were shown to selfadminister salsolinol. ${ }^{12}$ So, that compound is able to cause addiction because of some morphine-like effects. The study on whole-cell patch-clamps of rat brain ${ }^{13}$ confirmed that the mechanism of action of salsolinol is associated with stimulation of mu-opioid receptors. However, it is currently unknown whether morphine-like effects are caused by salsolinol itself or by one of its metabolites. Moreover, recent studies are focused on differences between two salsolinol enantiomers (R-salsolinol and S-salsolinol). Interestingly, R-salsolinol demonstrates morphine-like effects, while S-salsolinol does not. ${ }^{12}$ Other acetaldehyde conjugates with monoamines (at least, with serotonin, tryptamine, tryptophan) are biodegraded in the similar way to salsolinol, ${ }^{10}$ and they can form similar enantiomers. Their structures seem to have much more in common with morphine structure than the structure of salsolinol (they all have three rings, instead of two).

In animal models, it was shown that acetaldehyde intake usually produces stronger reinforcement than ethanol intake. ${ }^{11}$ Voluntary selfadministration of acetaldehyde is established in rats easier than that of ethanol. In low doses acetaldehyde causes effects similar to those of addictive drugs (stimulation and reinforcement), but in high doses acetaldehyde induces amnesia, ataxia, and loss of consciousness. ${ }^{9}$ There is even a specific term "acetaldehydism" that highlights that the majority of consequences of the high dose ethanol intake are due to 
acetaldehyde effects, while the majority of consequences of the low dose ethanol intake should be due to the action of $\mathrm{C}_{2} \mathrm{H}_{5} \mathrm{OH}$ molecule itself.

The aim of the current study was to perform molecular docking of four acetaldehyde conjugates with monoamines and their derivatives to the mu-opioid receptor structure and search for the most probable carriers of alcohol morphine-like effects.

In experiments performed in vivo $o^{12}$ or ex vivo ${ }^{13}$ injected compounds are not protected from the natural biodegradation processes. Because of this reason, there is a need for molecular docking experiments to find out whether the observed effects are due to the compound itself or due to one of its metabolites. The methods of molecular docking should be strong enough to answer this question. In our study the Docking Server (www.dockingserver.com) ${ }^{14,15}$ showed the different patterns of binding for enantiomers of N-methyl-salsolinol. This finding is consistent with the fact that R-salsolinol (according to our results, due to its $\mathrm{N}$-methylated derivative) but not $\mathrm{S}$-salsolinol is the transmitter of the morphine-like effect. ${ }^{12}$ Interestingly, N-methylR-salsolinol was also shown to impair dopamine and acetylcholine balance in rat brain even more than R-salsolinol. ${ }^{16}$ Current in silico study showed that $\mathrm{N}$-methylated and dehydrogenated derivative of the tryptophan conjugate with acetaldehyde should also be a strong muopioid agonist. Especially interesting biological aspects of this study are in the comparison of the results obtained with the Docking Server (which allows flexibility of ligands and does not allow flexibility of interacting amino acids), and the FlexX method which allows for flexibility of interacting amino acids.

\section{Materials and methods}

In this bioinformatic work, we used the recently published 3D structure of mouse mu-opioid receptor $(5 \mathrm{C} 1 \mathrm{M}) .{ }^{17}$ There are just 3 amino acid substitutions between mouse and human mu-opioid receptors. Rigid molecular docking has been performed with the help of the Docking Server (www.dockingserver.com). ${ }^{14,15}$ Flexible molecular docking has been performed with the help of the FlexX method $^{18}$ from the LeadIt suit. ${ }^{19}$ Using these powerful algorithms, we characterized possible interactions between mu-opioid receptor structure and the set of compounds: morphine, naloxone, dopamine, serotonin, tryptamine, and tryptophan. However, the main purpose of this study was to perform molecular docking with the mu-opioid receptor for the products of dopamine, serotonin, tryptamine, and tryptophan conjugation with acetaldehyde, as well as their metabolites: N-methylated and dehydrogenated ones. We considered $\mathrm{S}$ - and R-enantiomers for conjugates with acetaldehyde and their $\mathrm{N}$-methylated derivatives. 3D structures of the compounds were either downloaded from the PubChem (https://pubchem.ncbi.nlm.nih.gov/) database, or (in the case of their absence in the PubChem) drawn in the special editor program available via Docking Server web page. For each receptor-ligand pair, we performed 200 runs of docking ( 2 times with 100 runs) using the Docking Server. FlexX method generated several hundred poses for each compound. Then the average energy of binding $(\Delta \mathrm{G})$ and the probability of hydrogen bond formation between the ligand and the receptor have been calculated. Levels of average free energies have been compared by the two-tailed t-test, probabilities of hydrogen bond formation have been compared by the Chi-square test.

\section{Results}

\section{Docking of morphine and naloxone to mu-opioid receptor}

Using the Docking Server we performed docking of morphine and naloxone to the mu-opioid receptor. As it was expected, results for the agonist (morphine) and antagonist (naloxone) are different. The average free energy of binding $(\Delta \mathrm{G})$ for 200 runs of docking is equal to $-4.14 \pm 0.01 \mathrm{kcal} / \mathrm{mol}$ for morphine and $-4.61 \pm 0.14 \mathrm{kcal} / \mathrm{mol}$ for naloxone. The difference between those two average free energies is significant $(\mathrm{P}<0.001)$. This in silico experiment seems to be in agreement with the fact that naloxone can reversibly stop the action of morphine.

The set of amino acid residues involved in interactions with morphine in current docking sessions is in agreement with the known facts about the opioid binding pocket. ${ }^{17}$ Amino acid residues binding naloxone are usually the same as those in morphine dockings. As one can see in Figure 1, molecules of morphine and naloxone being mutually structurally related to each other usually demonstrate different orientations in the cavity between transmembrane helices. One of the possible reasons for the observed differences in the positions of the two molecules is in the hydrophobic contacts between the hydrophobic unsaturated "tail" $(-\mathrm{CH}=\mathrm{CH} 2)$ of naloxone molecule and such hydrophobic residues as Ile144 (Figure 1b). Morphine molecule lacks that hydrophobic tail (Figure 1a).
A

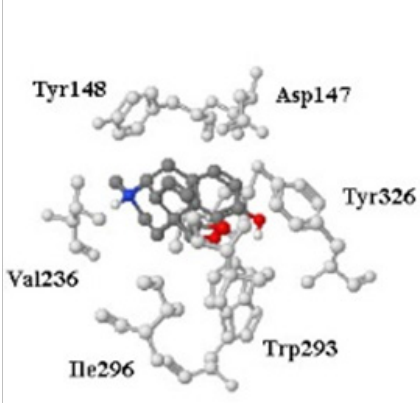

C

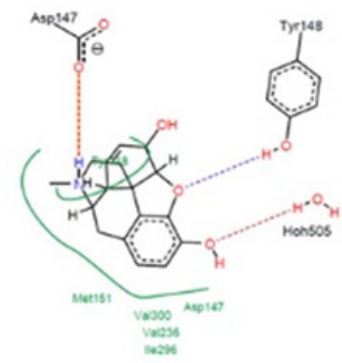

B

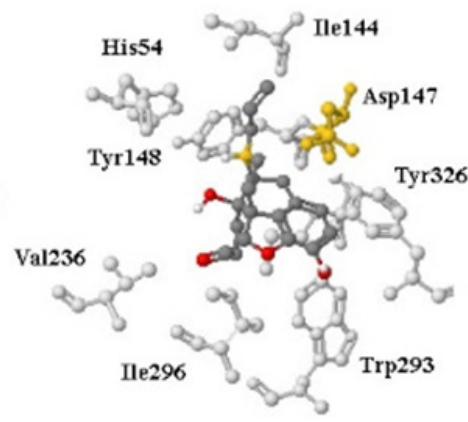

D

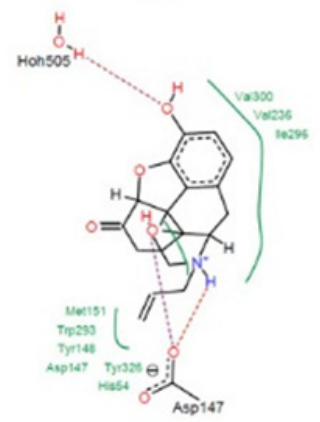

Figure I Rigid docking of morphine (a) and naloxone (b) to the structure of the mu-opioid receptor $(5 \mathrm{CIM})$. The most probable positions are shown. Aspl47 residue making a hydrogen bond to naloxone is shown in yellow. Best poses of the flexible docking of morphine (c) and naloxone (d) to the structure of the mu-opioid receptor $(5 \mathrm{CIM})$ contain information on hydrogen bonds (dotted lines) and hydrophobic interactions (green lines).

Different orientations cause changes in types of bonds with the same amino acid residues. Indeed, naloxone molecule makes hydrogen bonds to Asp147 and/or Tyr326 in 106 out of 200 docking runs. In contrast, there were just 14 out of 200 docking runs with morphine in which there was hydrogen bond between ligand and Tyr148. Interestingly, numerous hydrogen bonds with receptor are usually predicted for both ligands if we use flexible docking $(98 \%$ of poses for morphine and $95 \%$ of poses for naloxone contain at least one 
hydrogen bond). As one can see in Figure 1c, there are two hydrogen bonds between the protein and morphine molecule (with Asp147 and Tyr148) predicted by the flexible docking algorithm. Rigid (Figure 1c) and flexible docking (Figure 1d) algorithms both predicted that Asp147 makes a hydrogen bond with naloxone.

In vivo both morphine and naloxone should make hydrogen bonds with mu-opioid receptor since the formation of such bonds is usually thermodynamically favorable. Of course, the process of binding requires some flexibility of interacting amino acid residues. As a result, the binding of morphine leads to the rearrangement of the structure of mu-opioid receptor. Those rearrangements result in the signal transduction and the removal of the receptor from the membrane. ${ }^{20}$ Interactions with naloxone should not cause such drastic rearrangements of the architecture of the receptor.

In silico we can observe that morphine molecule usually cannot make a hydrogen bond with any oxygen or nitrogen atom from the cavity of the receptor if amino acid residues are rigid. Hydrophobic contacts, polar and aromatic interactions drive the position of morphine in the docking area. In contrast, naloxone molecule in more than a half runs finds the way to make a hydrogen bond.

Taken together, we can assume that because of some specific features of the structure of a ligand it may fit well to the docking area, make a hydrogen bond(s) with it in silico, and cause a little rearrangement in the architecture of the receptor in vivo. Such ligands should behave as antagonists. Compounds of a different structure may not fit well to the docking area, do not make hydrogen bonds with it during rigid docking in silico, and cause a substantial rearrangement in the architecture of the receptor in vivo to make hydrogen bonds. These types of compounds should behave as agonists.

So, the frequency of hydrogen bond formation during rigid docking experiments may be a criterion for the prediction of the way of ligand's action. Molecules that do not make (or rarely make) hydrogen bonds with mu-opioid receptor 3D structure in rigid docking experiments should behave as agonists, while molecules which usually make hydrogen bonds with the receptor should behave as antagonists.

\section{Docking of the dopamine and acetaldehyde conjugates and their derivatives to the mu-opioid receptor}

The product of dopamine and acetaldehyde conjugation is known under the name salsolinol. ${ }^{12}$ That compound has some structural similarities with morphine, as well as with naloxone. According to the docking results, S-salsolinol makes hydrogen bonds just with Asp147, just with Tyr326, or with both of them in all the 200 runs of the Docking Server. The average $\Delta \mathrm{G}$ for those 200 runs is $-5.72 \mathrm{kcal} /$ mol. unexpectedly, the results for R-salsolinol are very similar: hydrogen bond with Asp147 can be found in all the 200 runs, while $\Delta \mathrm{G}$ is significantly lower $(-6.87 \mathrm{kcal} / \mathrm{mol})$.

The free energy of binding for both isomers of salsolinol is really lower $(\mathrm{P}<0.001)$ than the one for the pure dopamine with mu-opioid receptor $(-5.28 \pm 0.03 \mathrm{kcal} / \mathrm{mol})$. Dopamine also makes hydrogen bonds with the antagonist containing structure in $100 \%$ of cases. So, conjugation of dopamine with acetaldehyde really enhances its ability to bind mu-opioid receptor. However, both dopamine and salsolinol isomers behave like antagonists and not agonists of that receptor.

In different in vivo experiments, it was shown that salsolinol injection promotes morphine-like symptoms in rats. ${ }^{12}$ It is also known that salsolinol and other conjugates of acetaldehyde and neurotransmitters are methylated and then dehydrogenated by enzymes involved in their degradation. ${ }^{10}$ Methylation happens at the nitrogen atom of salsolinol making this part of the molecule identical to the one from morphine (Figure 2). The following dehydrogenation happens at the same nitrogen atom. According to the results of docking of those two salsolinol derivatives to the structure of muopioid receptor, modifications of salsolinol decrease the affinity to the receptor but increase the probability of morphine-like action.

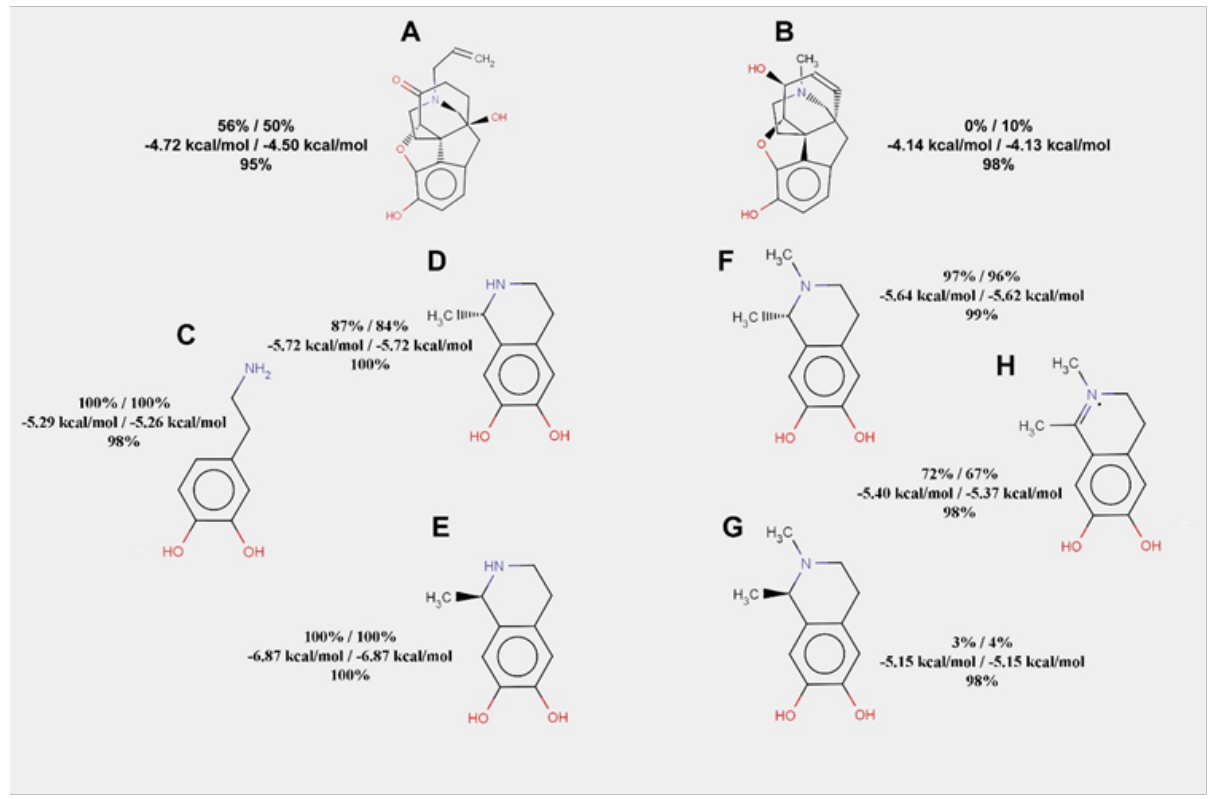

Figure 2 The results of dopamine, its conjugates with acetaldehyde, and their derivatives docking to the mu-opioid receptor. Frequencies of hydrogen bond formation (first line) and average free energies of binding (second line) are given for two rigid docking experiments (I 00 runs in each). Frequency of hydrogen bond formation is also given for flexible docking (third line) for each of the compounds listed below

a. Naloxone; b. Morphine; c. dopamine; d. S-isomer of the dopamine conjugate with acetaldehyde; e. R-isomer of the dopamine conjugate with acetaldehyde; $\mathbf{f}$. $\mathrm{N}$-methylated S-isomer of the dopamine conjugate with acetaldehyde; $\mathbf{g}$. N-methylated R-isomer of the dopamine conjugate with acetaldehyde; $\mathbf{h}$. N-methylated and dehydrogenated dopamine conjugate with acetaldehyde. 
For the methylated S-salsolinol, the frequency of hydrogen bond formation is 193 out of 200 . For the methylated R-salsolinol hydrogen bond has been found just in 7 out of 200 docking runs. For the methylated and dehydrogenated salsolinol the frequency of hydrogen bond formation is 139 out of 200. It means that the last compound (the methylated and dehydrogenated conjugate of acetaldehyde and dopamine) is not as good of a candidate for the alcohol morphine-like effect transduction as the methylated R-salsolinol.

The results of the Docking Server show that the known phenomenon of mu-opioid stereospecificity relative to salsolinol ${ }^{12}$ is linked to its $\mathrm{N}$-methylated derivative rather than to salsolinol itself. N-methyl-Rsalsolinol behaves like morphine analog in case if we perform rigid docking experiments with the $5 \mathrm{C} 1 \mathrm{M}$ structure. Indeed, in Figure 3A one can see that both Tyr326 and Asp147 are making hydrogen bonds to the nitrogen atom of N-methyl-S-salsolinol according to the best result of rigid docking. The most frequent result of the same rigid docking for R-methyl-salsolinol (Figure 3B) shows that nitrogen atom is situated far from Asp147 and Tyr326 residues.
A

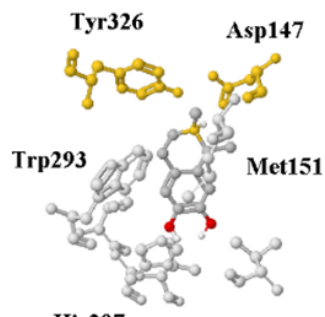

His297

C

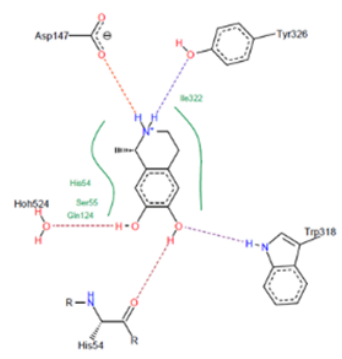

B

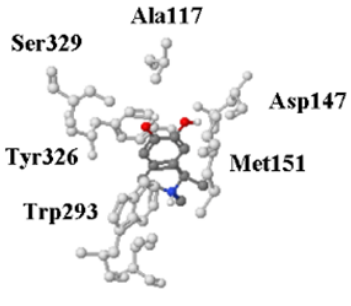

D

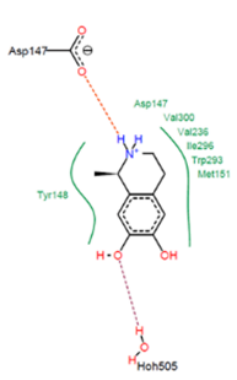

Figure 3 Rigid docking of N-methyl-S-salsolinol (a) and N-methyl-R-salsolinol (b) to the structure of the mu-opioid receptor (5CIM). The most probable positions are shown. Asp I 47 and Tyr 326 residues making a hydrogen bond to $\mathrm{N}$-methyl-S-salsolinol are shown in yellow. Best poses of the flexible docking of N-methyl-S-salsolinol (c) and N-methyl-R-salsolinol (d) to the structure of the mu-opioid receptor (5CIM) contain information on hydrogen bonds (dotted lines) and hydrophobic interactions (green lines).

Dehydrogenation of both N-methyl-S-salsolinol and N-methyl$\mathrm{R}$-salsolinol leads to the production of the same compound. This process does not affect the affinity to the receptor, but it decreases the probability of the morphine-like action for N-methyl-R-salsolinol.

Flexible docking predicts the formation of hydrogen bonds with the receptor for each of the abovementioned compounds (almost in every pose). Interestingly, as one can see in Figure 3C, the best pose for N-methyl-S-salsolinol has four hydrogen bonds with amino acid residues of the receptor (Asp147, Tyr326, Trp318, His54), while the best pose for N-methyl-R-salsolinol (Figure 3D) has just one hydrogen bond with the receptor (Asp147). Summarizing this section, we can conclude that $\mathrm{N}$-methyl-R-salsolinol has the strongest morphine-like potential of action for mu-opioid receptors.

\section{Docking of the serotonin and acetaldehyde conjugates and their derivatives to the mu-opioid receptor}

Serotonin and its conjugate with acetaldehyde demonstrate naloxone-like behavior in docking experiments with the structure of mu-opioid receptor. The probability of hydrogen bond formation is equal to $100 \%$ for serotonin, for both stereoisomers of its conjugate with acetaldehyde, for S-isomer of the methylated conjugate, and for the dehydrogenated form of the last one (Figure 4). For R-isomer of the methylated conjugate, the frequency of hydrogen bonds formation in rigid docking is just a little lower $(98.5 \%)$. Flexible docking also gives a high probability of hydrogen bonds formation for abovementioned compounds. However, some poses (less than 14\% for methylated derivatives) have no hydrogen bonds.

Among derivatives of serotonin and acetaldehyde conjugates we did not find as potent mu-opioid receptor agonists as among derivatives of dopamine and acetaldehyde conjugates.

\section{Docking of the tryptamine and acetaldehyde conjugates and their derivatives to the mu-opioid receptor}

Tryptamine, its conjugate with acetaldehyde, and its derivatives show strong naloxone-like behavior. For all of the compounds there were no rigid docking results without hydrogen bonds between the ligand and the mu-opioid receptor structure. Even in flexible docking, the percent of poses without hydrogen bonds was just a little lower than $100 \%$ (from 96 to $99 \%$ ). So, tryptamine acetylation products (Figure 5) seem to be worse candidates for morphine-like effects of alcohol intake than serotonin acetylation products.

\section{Docking of the tryptophan and acetaldehyde conjugates and their derivatives to the mu-opioid receptor}

It is known that inclusion of tryptophan increases agonist-like effects of synthetic peptides, which are able to bind mu-opioid receptors. ${ }^{21-23}$ However, tryptophan itself shows $100 \%$ probability of hydrogen bond formation with the receptor in rigid docking. The same thing can be said about its conjugates with acetaldehyde and their methylated forms (Figure 6). Surprisingly, dehydrogenation of the N-methylated conjugate of tryptophan and acetaldehyde highly increases its ability to imitate morphine: just 9 out of 200 docking runs resulted in hydrogen bonds formation between that ligand and mu-opioid receptor.

Even in flexible docking, the percent of poses with hydrogen bonds for dehydrogenated N-methylated conjugate of tryptophan and acetaldehyde $(61 \%)$ is the lowest one (among all the compounds tested in this study). We may hypothesize that binding of methylated and dehydrogenated conjugate of tryptophan and acetaldehyde may require drastic changes in the architecture of the receptor which often cannot be modeled even by the flexible docking. According to the docking results, one may suspect that just dopamine and tryptophan (and not serotonin and tryptamine) give rise to the compounds acting as agonists of mu-opioid receptors. 


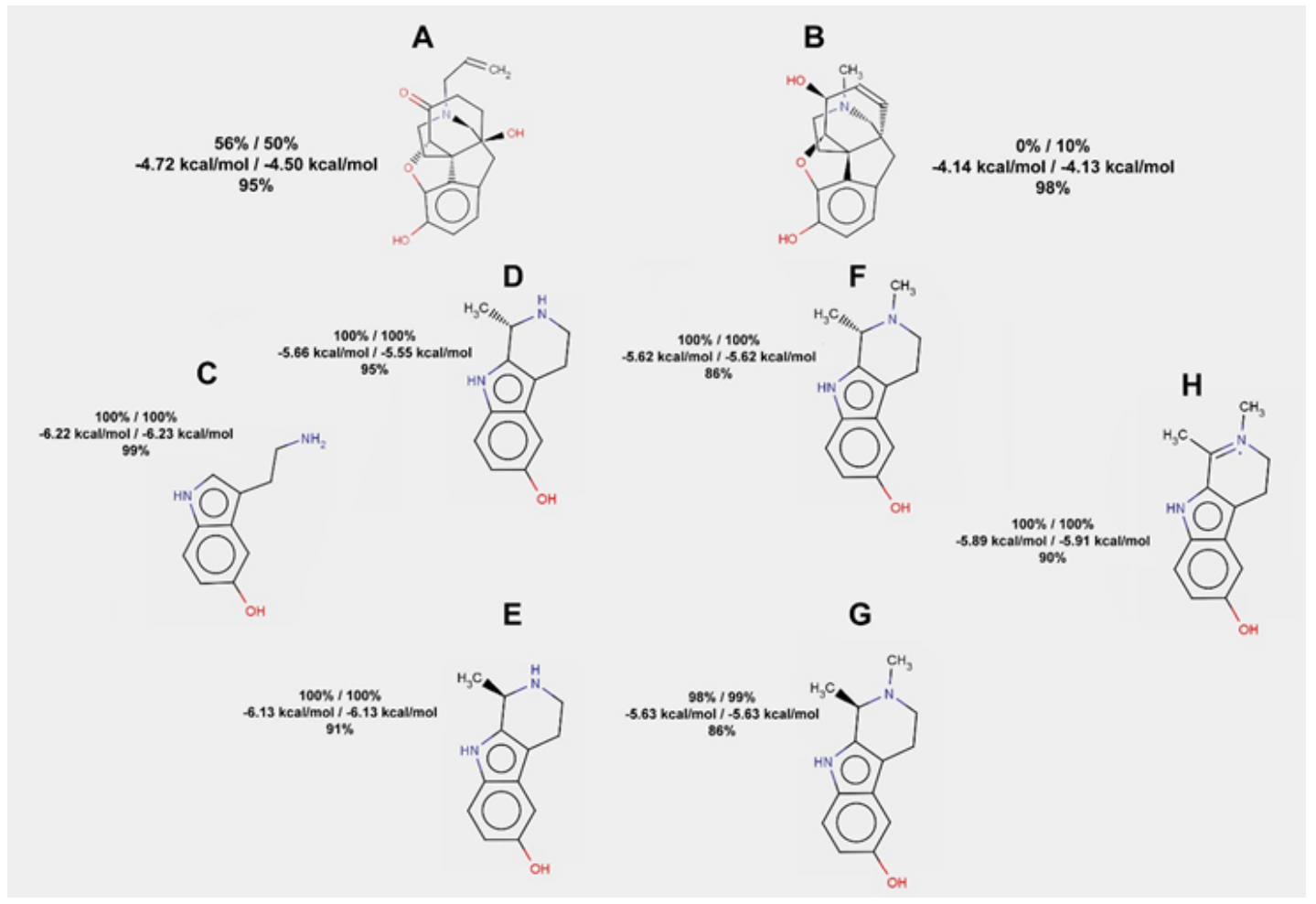

Figure 4 The results of serotonin, its conjugates with acetaldehyde, and their derivatives docking to the mu-opioid receptor. Frequencies of hydrogen bond formation (first line) and average free energies of binding (second line) are given for two rigid docking experiments ( 100 runs in each). Frequency of hydrogen bond formation is also given for flexible docking (third line) for each of the compounds listed below

a. Naloxone; b. Morphine; c. serotonin; d. S-isomer of the serotonin conjugate with acetaldehyde; e. R-isomer of the serotonin conjugate with acetaldehyde; f. $\mathrm{N}$-methylated S-isomer of the serotonin conjugate with acetaldehyde; $\mathbf{g}$. N-methylated $\mathrm{R}$-isomer of the serotonin conjugate with acetaldehyde; $\mathbf{h}$. $\mathrm{N}$-methylated and dehydrogenated serotonin conjugate with acetaldehyde.

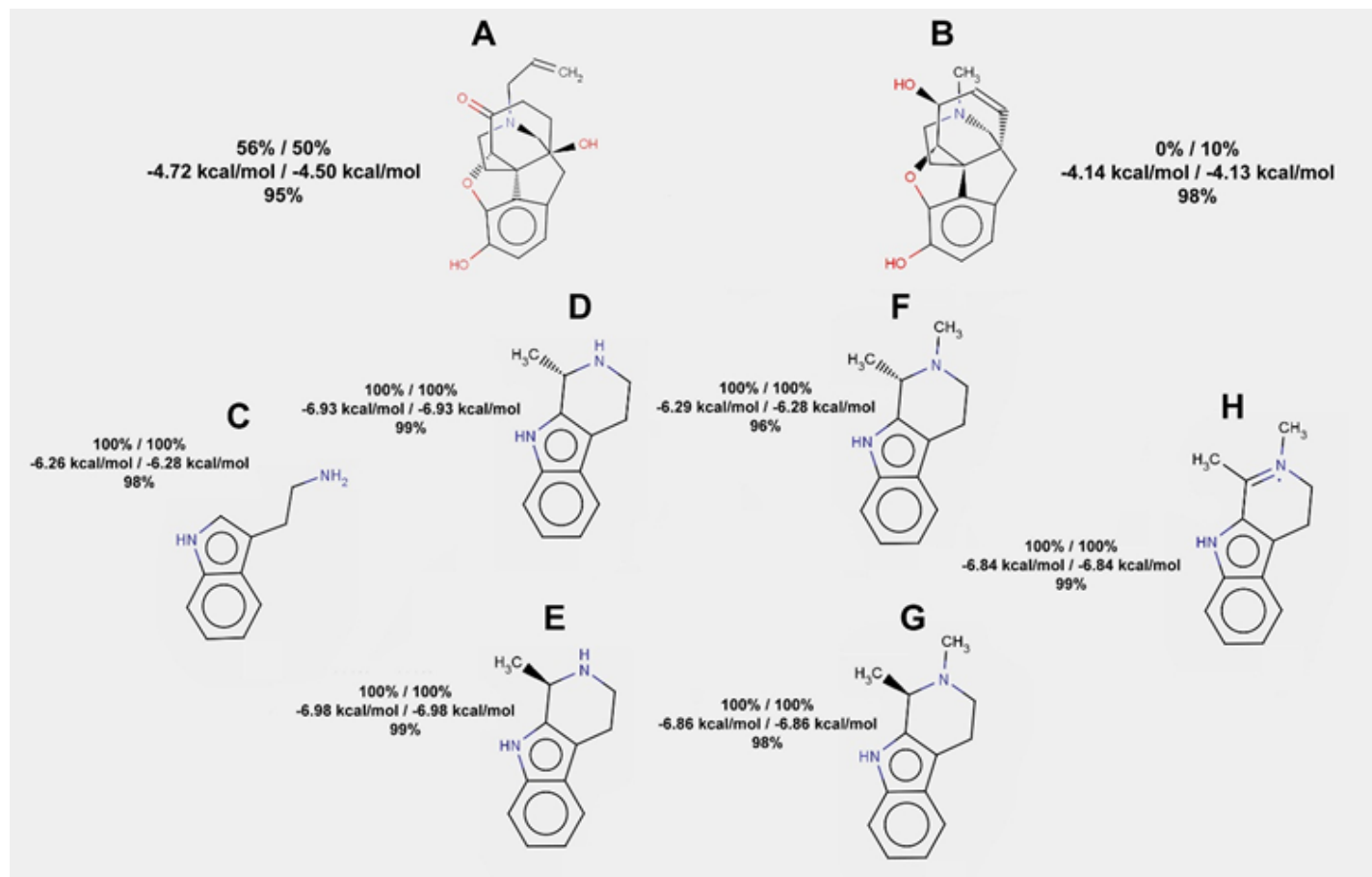

Figure $\mathbf{5}$ The results of tryptamine, its conjugates with acetaldehyde, and their derivatives docking to the mu-opioid receptor. Frequencies of hydrogen bond formation (first line) and average free energies of binding (second line) are given for two rigid docking experiments ( 100 runs in each). Frequency of hydrogen bond formation is also given for flexible docking (third line) for each of the compounds listed below

a. Naloxone; b. Morphine; c. Tryptamine; d. S-isomer of the tryptamine conjugate with acetaldehyde; e. R-isomer of the tryptamine conjugate with acetaldehyde; f. $\mathrm{N}$-methylated $\mathrm{S}$-isomer of the tryptamine conjugate with acetaldehyde; $\mathbf{g}$. $\mathrm{N}$-methylated R-isomer of the tryptamine conjugate with acetaldehyde; $\mathbf{h}$. $\mathrm{N}$-methylated and dehydrogenated tryptamine conjugate with acetaldehyde. 


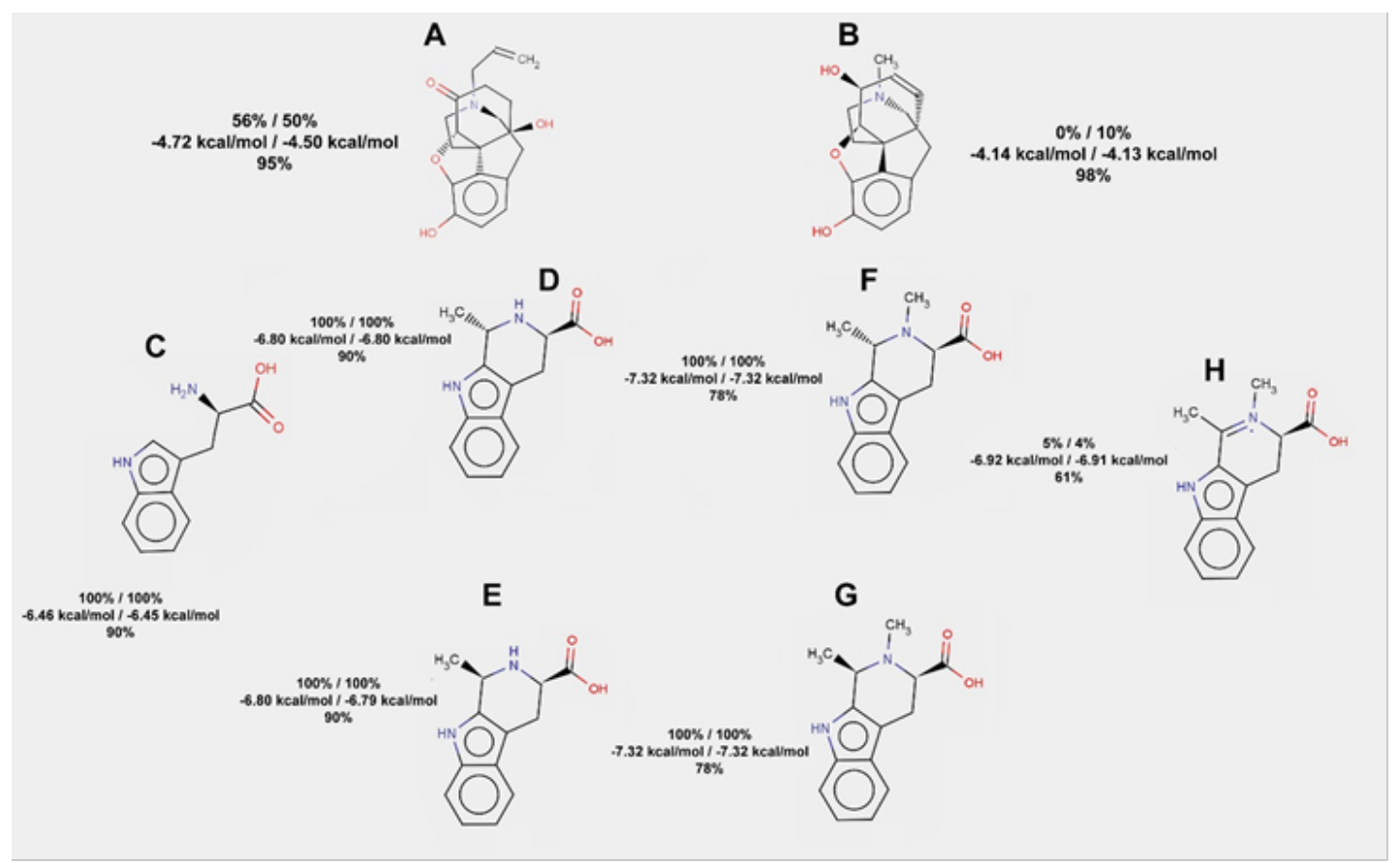

Figure 6 The results of tryptophan, its conjugates with acetaldehyde, and their derivatives docking to the mu-opioid receptor. Frequencies of hydrogen bond formation (first line) and average free energies of binding (second line) are given for two rigid docking experiments (I00 runs in each). Frequency of hydrogen bond formation is also given for flexible docking (third line) for each of the compounds listed below

a. Naloxone; b. Morphine; c. Tryptophan; d. S-isomer of the tryptophan conjugate with acetaldehyde; $\mathbf{e}$. R-isomer of the tryptophan conjugate with acetaldehyde; f. $\mathrm{N}$-methylated S-isomer of the tryptophan conjugate with acetaldehyde; $\mathbf{g}$. N-methylated R-isomer of the tryptophan conjugate with acetaldehyde; $\mathbf{h}$. $\mathrm{N}$-methylated and dehydrogenated tryptophan conjugate with acetaldehyde.

\section{Discussion}

Currently, bioinformatics is moving towards a new era. Results of many in silico experiments are not directly reproduced in vivo, while they give us additional information about modeled processes. That kind of information may bring new insights into understanding of the processes that are hard to study by any "wet" method. Regarding the current study, it is hard to claim that morphine does not make hydrogen bonds with amino acids from the mu-opioid receptor in vivo, but it really does not make them in the rigid molecular docking simulations. If naloxone makes those hydrogen bonds with mu-opioid receptor structure in more than a half runs, than the results of rigid docking cannot be ignored - they do contain some useful information. So, we can use exactly the Docking Server and exactly $5 \mathrm{C} 1 \mathrm{M} \mathrm{mu}$ opioid receptor structure to check whether a compound behaves like an agonist or antagonist of the receptor. This simulation is not a reflection of the real interactions between ligand and receptor, but it may be considered to be a kind of "in silico phenomenon" that we can use. So, such criterion as the probability of hydrogen bond formation between a ligand and the mu-opioid receptor in the molecular docking experiments may be used to discriminate between agonist-like and antagonist-like compounds. Based on this in silico phenomenon, we tested the ability of derivatives of the biogenic amines conjugation with acetaldehyde to act as mu-opioid receptor agonists.

We confirmed that conjugation of biogenic amines with acetaldehyde increases their affinities to mu-opioid receptor. We also confirmed that tryptophan and its conjugate with acetaldehyde demonstrate high affinity to that receptor. Indeed, many endogenic peptides acting as mu-opioid receptor agonists contain tryptophan. ${ }^{22,23}$ So, tryptophan and its conjugate with acetaldehyde may act as endogenous mu-opioid receptor antagonists, while the metabolite of the last compound (N-methylated and dehydrogenated one) may act as agonist. Probably, if the increase of tryptophan level in neural tissue happens after the alcohol intake (when acetaldehyde level is already high), it may cause stimulation of mu-opioid receptors and further establishment of the addiction to alcohol. On the other hand, if there is a high background level of tryptophan, it will occupy mu-opioid receptors in antagonist-like manner and block interactions of the receptors with agonist-like derivatives produced during the alcohol intake. Indeed, the usage of tryptophan in alcoholism treatment has some controversies. ${ }^{24,25}$ The dietary tryptophan enhancement selectively facilitates stress-induced increase in craving to alcohol in the cohort of high-hostile alcoholics, but not in low-hostile ones. ${ }^{24}$ A tryptophan-enriched diet helps binge-drinkers to cope with stress, while it facilitates an increase in the strong desire to consume alcohol in stressful conditions for non-binge drinkers. ${ }^{25}$

According to our results, morphine-like effects observed as a result of direct salsolinol injection ${ }^{12}$ should develop due to fast $\mathrm{R}$-salsolinol N-methylation and slow dehydrogenation. So, the efficiency of salsolinol methylation and dehydrogenation should play a significant role in the individual's behavior after the alcohol intake. Fast biodegradation (both N-methylation and dehydrogenation) of tryptophan conjugates with acetaldehyde should be one of the factors increasing the probability of the addiction to alcohol development. $\mathrm{N}$-methyl-R-salsolinol is known as a toxic agent inducing apoptosis in dopaminergic neurons. ${ }^{26}$ Moreover, that compound is able to inhibit type 1 monoamine oxidase..$^{27,28}$ So, N-methyl-R-salsolinol should partially block its own dehydrogenation by the type 1 monoamine oxidase. 
Antagonist binding by mu-opioid receptors should follow the common rule known for ligands and receptors: the binding increases stability of the complex. Hydrogen bonds between antagonist and receptor should stabilize the structure of the whole complex. From this point of view, the majority of compounds able to bind mu-opioid receptors should act as antagonists. Agonists of mu-opioid receptors must demonstrate some specific features: they have to bind receptors with a high affinity but allow forthcoming structural changes. Hydrophobic contacts may be more energetically favorable than hydrogen bonds. However, hydrophobic contacts allow much higher flexibility of the complex than hydrogen bonds. Literally, if there are no hydrogen bonds between ligand and receptor, transmembrane helices may easily slide relative to each other. The final result of such sliding should be the formation of a new ligand-receptor complex with hydrogen bonds. From this point of view, we can speculate that rigid docking predicts the transition state of the ligand-receptor complex, while flexible docking predicts the final state. If we assume that the final state of the complex is stabilized by hydrogen bonds between ligand and receptor, then their existence in the rigid docking results can be considered as an argument towards the statement that there will be no further changes in the architecture of the protein.

\section{Conclusion}

a. Naloxone forms hydrogen bonds with mu-opioid receptor structure $(5 \mathrm{C} 1 \mathrm{M})$ in the Docking Server simulations in more than a half runs, while morphine usually does not form them. This in silico phenomenon can be used as a criterion to distinguish agonists and antagonists of the mu-opioid receptor.

b. According to the abovementioned criterion, N-methyl-Rsalsolinol is a stereospecific mu-opioid receptor agonist. This compound should be responsible for morphine-like effects of ethanol, unlike R-salsolinol itself. Further biodegradation of N-methyl-R-salsolinol by dehydrogenation decreases the strength of its morphine-like effect.

c. According to the same criterion, the product of N-methylation and dehydrogenation of the tryptophan conjugate with acetaldehyde demonstrates properties of mu-opioid receptor agonist.

\section{Acknowledgements}

None.

\section{Conflict of interest}

The author declares no conflict of interest.

\section{References}

1. Davis TJ, deFiebre CM. Alcohol's actions on neuronal nicotinic acetylcholine receptors. Alcohol Res Health. 2006;29(3):179-185.

2. Aryal P, Dvir H, Choe S, et al. A discrete alcohol pocket involved in GIRK channel activation. Nat Neurosci. 2009;12(8):988-995.

3. Yevenes GE, Moraga-Cid G, Avila A, et al. Molecular requirements for ethanol differential allosteric modulation of glycine receptors based on selective G modulation. J Biol Chem. 2010;285(39):30203-30213.

4. Meera P, Olsen RW, Otis TS, et al. Alcohol and alcohol antagonistsensitive human GABAA receptors: tracking subunit incorporation into functional receptors. Mol Pharmacol. 2010;78(5):918-924.

5. Ren H, Salous AK, Paul JM, et al. Functional interactions of alcoholsensitive sites in the N-methyl-D-aspartate receptor M3 and M4 domains. J Biol Chem. 2008;283(13):8250-8257.
6. Lelevich SV, Khrustalev VV, Barkovsky EV, et al. The influence of ethanol on pyruvate kinases activity in vivo, in vitro, in silico. AJMBR. 2013;1(1):6-15.

7. Lelevich SV, Khrustalev VV, Barkovsky EV. Inhibition of rat muscle and liver phosphofructokinases by high doses of ethanol. Biochem Res Int. 2013;2013:495135.

8. Melis M, Carboni E, Caboni P, et al. Key role of salsolinol in ethanol actions on dopamine neuronal activity of the posterior ventral tegmental area. Addict Biol. 2015;20(1):182-193.

9. Quertemont E, Didone V. Role of acetaldehyde in mediating the pharmacological and behavioral effects of alcohol. Alcohol Research \& Health. 2006;29(4):258-265.

10. Deitrich R, Zimatkin S, Pronko S. Oxidation of ethanol in the brain and its consequences. Alcohol Research \& Health. 2006;29(4):266-273.

11. Israel Y, Quintanilla ME, Karahanian E, et al. The "first hit" toward alcohol reinforcement: role of ethanol metabolites. Alcohol Clin Exp Res. 2015;39(5):776-786

12. Quintanilla ME, Rivera-Meza M, Berríos-Cárcamo P, et al. (R)Salsolinol, a product of ethanol metabolism, stereospecifically induces behavioral sensitization and leads to excessive alcohol intake. Addict Biol. 2015;21(6):1063-1071.

13. Xie G, Hipólito L, Zuo W, et al. Salsolinol stimulates dopamine neurons in slices of posterior ventral tegmental area indirectly by activating $\mu-$ opioid receptors. J Pharmacol Exp Ther. 2012;341(1):43-50.

14. Huey R, Morris GM, Olson AJ, et al. A semiempirical free energy force field with charge-based desolvation. J Comput Chem. 2007;28(6):11451152.

15. Bikadi Z, Hazai E. Application of the PM6 semi-empirical method to modeling proteins enhances docking accuracy of AutoDock. $J$ Cheminform. 2009;1:15.

16. Zhu W, Wang D, Zheng J, et al. Effect of (R)-salsolinol and N-methyl(R)-salsolinol on the balance impairment between dopamine and acetylcholine in rat brain: involvement in pathogenesis of Parkinson disease. Clin Chem. 2008;54(4):705-712.

17. Huang W, Manglik A, Venkatakrishnan AJ, et al. Structural insights into $\mu$-opioid receptor activation. Nature. 2015;524(7565):315-321.

18. Kramer B, Rarey M, Lengauer T. Evaluation of the FlexX incremental construction algorithm for protein-ligand docking. Proteins. 1999;37(2):228-241.

19. Maass P, Schulz-Gasch T, Stahl M, et al. Recore: A fast and versatile method for scaffold hopping based on small molecule crystal structure conformations. J Chem Inf Model. 2007;47(2):390-399.

20. Zhang L, Zhao H, Qiu Y, et al. Src phosphorylation of micro-receptor is responsible for the receptor switching from an inhibitory to a stimulatory signal. J Biol Chem. 2009;284(4):1990-2000.

21. Xu H, Lu YF, Partilla JS, et al. Opioid peptide receptor studies, 11: involvement of Tyr148, Trp318 and His319 of the rat mu-opioid receptor in binding of mu-selective ligands. Synapse. 1999;32(1):23-28.

22. De Marco R, Bedini A, Spampinato S, et al. Synthesis of tripeptides containing D-Trp substituted at the indole ring, assessment of opioid receptor binding and in vivo central antinociception. J Med Chem. 2014;57(15):6861-6866.

23. Del Borgo MP, Blanchfield JT, Toth I. Internalisation of the mu-opioid receptor by endomorphin-1 and leu-enkephalin is dependent on aromatic amino acid residues. Bioorg Med Chem. 2008;16(8):4341-4346.

24. Nesic J, Duka T. Effects of stress on emotional reactivity in hostile heavy social drinkers following dietary tryptophan enhancement. Alcohol Alcohol. 2008;43(2):151-162. 
25. Nesic J, Duka T. Effects of stress and dietary tryptophan enhancement on craving for alcohol in binge and non-binge heavy drinkers. Behav Pharmacol. 2014;25(5-6):503-517.

26. Naoi M, Maruyama W, Akao Y, et al. Apoptosis induced by an endogenous neurotoxin, $\mathrm{N}-$-methyl(R)salsolinol, in dopamine neurons. Toxicology. 2000;153(1-3):123-141.

27. Naoi M, Maruyama W, Nagy GM. Dopamine-derived salsolinol derivatives as endogenous monoamine oxidase inhibitors: occurrence, metabolism and function in human brains. Neurotoxicology. 2004;25(12):193-204.
28. Yi H, Akao Y, Maruyama W, et al. Type A monoamine oxidase is the target of an endogenous dopaminergic neurotoxin, N-methyl(R) salsolinol, leading to apoptosis in SH-SY5Y cells. J Neurochem. 2006;96(2):541-549. 